An Improved Method of Mitigating Laser Induced Surface Damage Growth in Fused Silica Using a Rastered, Pulsed CO2 Laser

I. L. Bass, G. M. Guss, M. J. Nostrand, P. L. Wegner

November 15, 2010

SPIE Laser Damage

Boulder, CO, United States

September 26, 2010 through September 29, 2010 
This document was prepared as an account of work sponsored by an agency of the United States government. Neither the United States government nor Lawrence Livermore National Security, LLC, nor any of their employees makes any warranty, expressed or implied, or assumes any legal liability or responsibility for the accuracy, completeness, or usefulness of any information, apparatus, product, or process disclosed, or represents that its use would not infringe privately owned rights. Reference herein to any specific commercial product, process, or service by trade name, trademark, manufacturer, or otherwise does not necessarily constitute or imply its endorsement, recommendation, or favoring by the United States government or Lawrence Livermore National Security, LLC. The views and opinions of authors expressed herein do not necessarily state or reflect those of the United States government or Lawrence Livermore National Security, LLC, and shall not be used for advertising or product endorsement purposes. 


\title{
An Improved Method of Mitigating Laser Induced Surface Damage Growth in Fused Silica Using a Rastered, Pulsed $\mathrm{CO}_{2}$ Laser
}

\author{
Isaac L. Bass", Gabriel M. Guss, Michael J. Nostrand, and Paul J. Wegner \\ Lawrence Livermore National Security, LLC, \\ Lawrence Livermore National Laboratory, Livermore, CA
}

\begin{abstract}
A new method of mitigating (arresting) the growth of large (>200 $\mu \mathrm{m}$ diameter and depth) laser induced surface damage on fused silica has been developed that successfully addresses several issues encountered with our previously-reported ${ }^{5,6}$ large site mitigation technique. As in the previous work, a tightly-focused $10.6 \mu \mathrm{m} \mathrm{CO}_{2}$ laser spot is scanned over the damage site by galvanometer steering mirrors. In contrast to the previous work, the laser is pulsed instead of $\mathrm{CW}$, with the pulse length and repetition frequency chosen to allow substantial cooling between pulses. This cooling has the important effect of reducing the heat-affected zone capable of supporting thermo-capillary flow from scale lengths on the order of the overall scan pattern to scale lengths on the order of the focused laser spot, thus preventing the formation of a raised rim around the final mitigation site and its consequent down-stream intensification. Other advantages of the new method include lower residual stresses, and improved damage threshold associated with reduced amounts of redeposited material. The raster patterns can be designed to produce specific shapes of the mitigation pit including cones and pyramids. Details of the new technique and its comparison with the previous technique will be presented.
\end{abstract}

Keywords: Mitigation, surface damage, fused silica, $\mathrm{CO}_{2}$ laser, pulsed.

\section{INTRODUCTION}

Inertial confinement fusion (ICF) class laser systems will typically expose high-value, fused-silica optics to fluences near their damage threshold. Considerable work has been done to develop techniques to mitigate growth of damage sites which may be initiated on these optics. The most commonly used method at this time for mitigating the surface damage on fused silica optics employs a $10.6 \mu \mathrm{m}$ wavelength $\mathrm{CO}_{2}$ laser to melt or evaporate the damage ${ }^{1-7}$ (mitigation with a 4.6 $\mu \mathrm{m} \mathrm{CO}_{2}$ laser has also been demonstrated $\left.{ }^{8,9}\right)$. The $10.6 \mu \mathrm{m}$ radiation is effective because it is strongly absorbed within a few microns of the fused silica surface.

The surface shape modification produced by mitigation can lead to intensification of the laser light passing through it during laser operation. It is critical that the intensified light remain below the damage threshold of other downstream optical surfaces in the laser system. If mitigation involves evaporation, fused silica vapor or particles re-deposited on the surface can damage on subsequent exposure to high fluence laser light. Avoiding this problem is another requirement of the mitigation process. Rapid cooling of the fused silica melted during mitigation leads to residual stress. If this stress is too high, subsequent laser damage near the stressed region can cause long surface cracks to open that are not amenable to mitigation. It is therefore important to avoid excessive residual stress from the mitigation process.

Here we describe a new technique of evaporative mitigation that corrects problems of downstream intensification, redeposited material, and residual stress that occurred with the previous evaporative technique that we have reported.,6 The primary advantage of evaporative mitigation is its scalability to large damage sites with lateral dimensions $>200 \mu \mathrm{m}$ and subsurface cracks deeper than $200 \mu \mathrm{m}$. In this report we will emphasize its application to such large damage sites.

\section{THE PREVIOUS EVAPORATIVE MITIGATION TECHNIQUE}

The previous evaporative mitigation technique is illustrated schematically in Fig. 1. In the first step, the laser damage is completely evaporated by rapidly scanning the tightly focused laser spot over the damage site with the laser operating

\footnotetext{
* bass1@1lnl.gov; mailstop: L-592; phone 925-423-8389; fax 925-422-5099
} 
$\mathrm{CW}$. The bright circular ring indicates the location of the raised-rim around the mitigation pit as shown in Fig. 2a. The rim is the result of thermo-capillary flow under large temperature gradients (the Marangoni $\mathrm{effect}^{10}$ ). The outer portions

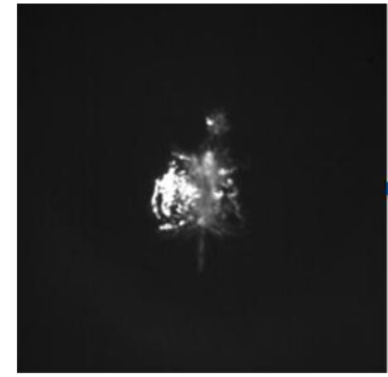

Original damage

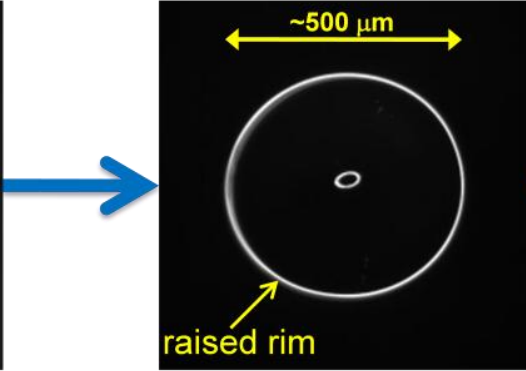

Evaporation of damage

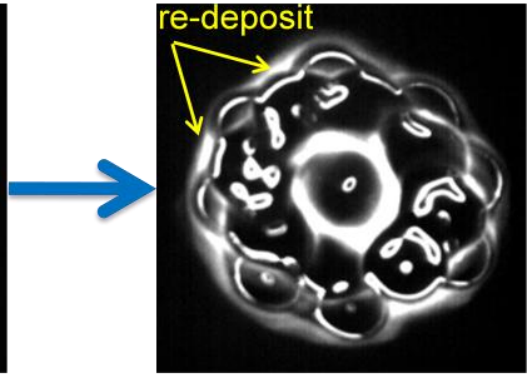

Dimpling of rim
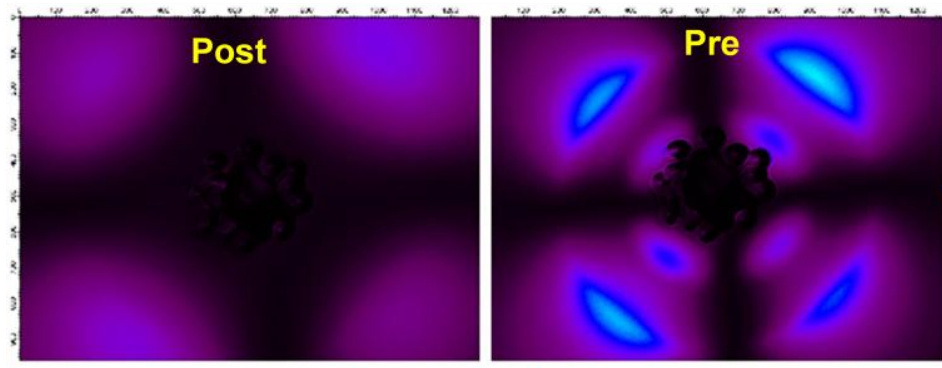

Anneal stress

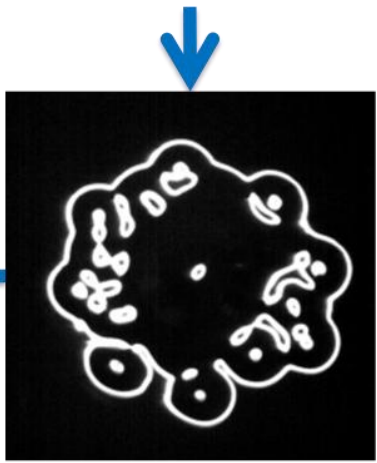

Melt re-deposit

Figure 1. Optical microscope images of the steps involved in the previous evaporative mitigation technique. Crossed-polarizers placed before and after the sample in the last two images reveal the birefringence pattern caused by stress.

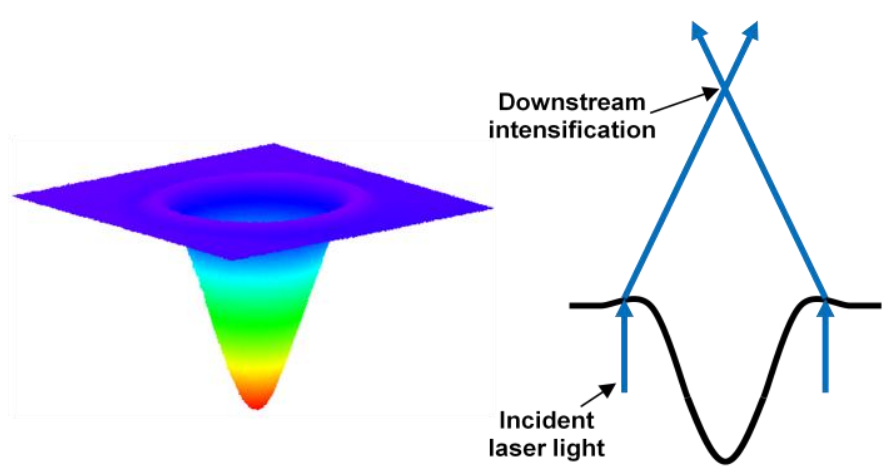

(a) (b)

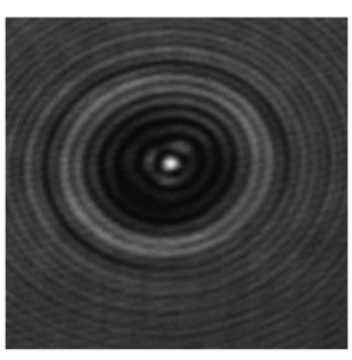

(c)

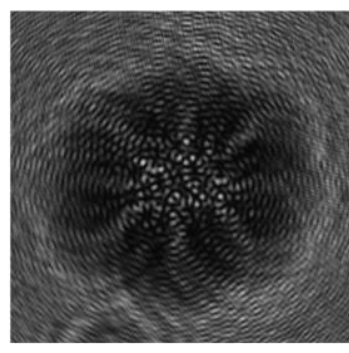

(d)

Figure 2. (a) Illustration of the raised rim around the mitigation pit after evaporation of the damage. (b) Representation of the focusing of light refracted from the outer portions of the raised rim. (c) The on-axis hot-spot caused by the light refracted from the outer portions of the raised rim. (d) Break-up and reduction of the hot-spot by "dimpling" the raised rim.

of the rim refract light toward the center as illustrated in Fig. 2b. This refracted light focuses downstream to create an intense, on-axis hot-spot shown in Fig. 2c that could damage other optical surfaces.

To reduce the intensity of the on-axis hot-spot, the circular symmetry of the raised rim is broken-up by a series of randomly placed, small evaporation "dimples" as seen in Fig. 1. The resulting downstream intensification pattern is shown in Fig. 2d. However, "dimpling" generates a significant amount of re-deposit surrounding the mitigation site as 
indicated by the gray areas in Fig. 1 and shown in the left micrograph of Fig. 3. To avoid subsequent laser damage caused by this re-deposit, it is re-melted at temperatures below the evaporation temperature with an enlarged laser spot (right micrograph of Fig. 3). The rapid cooling after this process in turn leads to high residual stresses that must be annealed with yet another, larger laser spot as shown in Fig. 1.
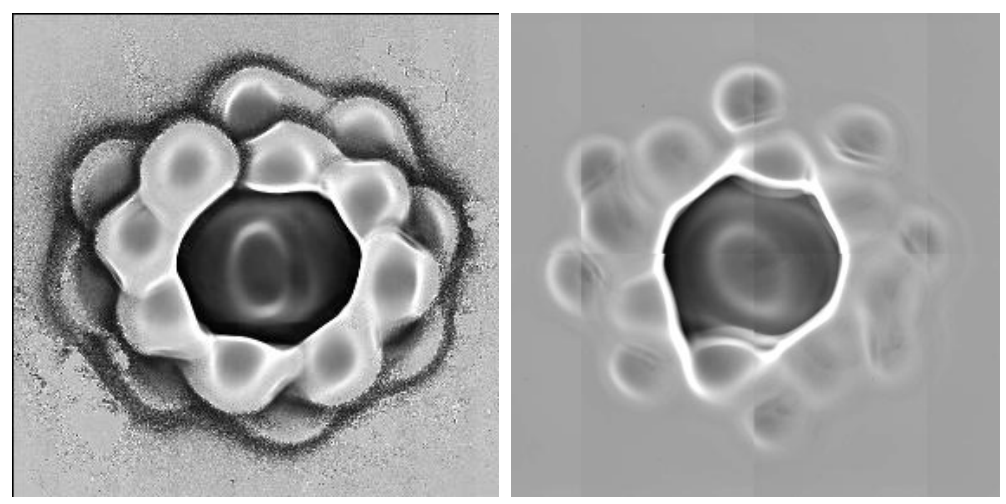

Figure 3. Optical microscope images of the re-deposit surrounding the mitigation pit caused by "dimpling" of the rim before (left) and after (right) re-melting.

Thus, this evaporative mitigation technique involved 4 steps with optical configuration changes for the last 2 steps. In addition, it was somewhat sensitive to the position of the focusing lens and laser power, especially in the re-melting step where insufficient melting does not increase the damage threshold of the re-deposit enough, and excessive melting regenerates high downstream intensification. For these reasons it was of questionable suitability for an optical damage mitigation facility to treat ICF-class optics.

\section{THE NEW EVAPORATIVE MITIGATION TECHNIQUE}

As with the previous technique, the new technique ${ }^{\dagger}$ also involves scanning the tightly focused laser spot $\left(\sim 130 \mu \mathrm{m} 1 / \mathrm{e}^{2}\right.$ diameter) over the mitigation site using galvanometer driven scanning mirrors. One critical difference is that the laser is now operated in a pulsed mode as opposed to the $\mathrm{CW}$ mode used previously. Pulse lengths are typically less than $50 \mu \mathrm{s}$. The power during the pulse is roughly 3 times the $\mathrm{CW}$ power used in the previous method. The result is that evaporation is much more rapid taking place at temperatures estimated to be between $4000 \mathrm{~K}$ and $5000 \mathrm{~K}$ compared to $3000 \mathrm{~K}$ in the previous technique. The other critical difference is that the pulse repetition frequency (PRF) is selected to allow substantial cooling between pulses. ${ }^{\ddagger}$ The result is that thermo-capillary flow is limited in space to dimensions of the order of the laser spot, and times of the order of the pulse duration. By contrast, areas comparable to the entire scan pattern are heated to melting temperatures in the previous technique. This resulted in thermo-capillary flow over these large areas during the entire evaporation process lasting several hundred milliseconds.

As will be seen below, the spatially and temporally limited thermo-capillary flow of the new technique leads to a much smaller raised rim eliminating the need for "dimpling" The very high evaporation rates resulting from the high evaporation temperatures lead to minimal re-deposit eliminating the need for re-melting. The lower average power, $\sim 1$ $\mathrm{W}$ compared to $>10 \mathrm{~W}$ previously, leads to negligible residual stress eliminating the need for annealing. Thus, the new process involves only the single evaporation step compared to the 4 steps needed previously with their associated configuration changes. This makes the new process much more suitable for a production facility. An additional and very important benefit is the ability to control the final shape on scales larger than the laser spot size.

A schematic diagram of the mitigation system is shown in Fig. 4. A $100 \mathrm{~W}, 10.6 \mu \mathrm{m}$ wavelength, commercial, RFexcited, waveguide, $\mathrm{CO}_{2}$ laser is operated at $10 \mathrm{kHz}$ with a $50 \%$ duty factor. A commercial acousto-optic modulator

\footnotetext{
${ }^{\dagger}$ U.S. Patent Application No. 12/818094

\# The calculated thermal diffusion time for fused silica over the laser spot dimensions is of the order of the $500 \mu$ s time between pulses at $2 \mathrm{kHz}$.
} 
operated synchronously at a sub-multiple of the $10 \mathrm{kHz}$ deflects pulses at the peaks of the laser output waveform into the first order diffracted beam. Sub-multiple PRFs range between $250 \mathrm{~Hz}$ to $2 \mathrm{kHz}$ depending on the particular mitigation shape and size desired. A quarter waveplate converts the linear polarization to circular in order to eliminate asymmetries in the mitigation shape resulting from polarization effects. The beam is then expanded to $\sim 20 \mathrm{~mm}$ by a commercial beam expander before entering the 2-axis, commercial galvanometer scanner. The output of the scanner is focused onto the fused silica optic by a ZnSe lens. An optional vacuum nozzle may be used to control the evaporated material.

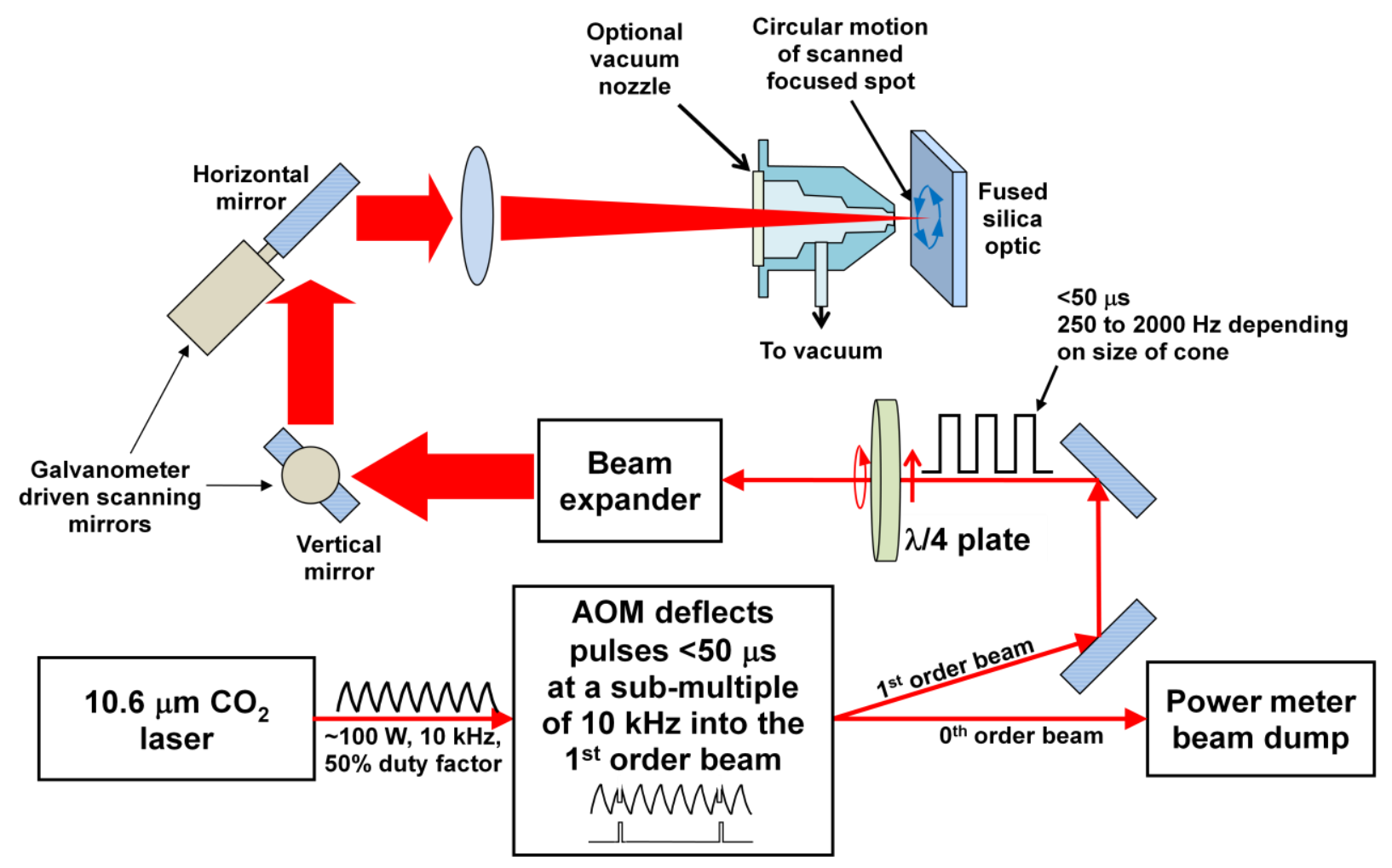

Figure 4. Schematic diagram of the mitigation system.

We have found that a conical shape is best suited in most instances for mitigation. Reasons include minimization of the mitigation site footprint and evaporated material, an even perimeter, and the decrease in intensity of the light refracted from the walls with propagation distance. To generate the cone, a scan pattern like the one illustrated schematically in Fig. 5a is used. The pattern consists of a series of concentric circles. The initial radius is the cone radius. After completion of each circle the radius is decreased by an amount $\delta$ in the figure (typically $20 \mu \mathrm{m}$ ). This continues until the radius becomes zero. Then the radius starts increasing until it is $\delta$ less than the initial cone radius. Then the decreasing radius pattern starts again from the cone radius minus $\delta / 2$. This sequence continues until the ever decreasing starting radius reaches zero. The scan direction around the circles alternates between clockwise and counterclockwise to reduce any systematic effects on the shape of the mitigation site surface. A measured surface profile of a typical $2 \mathrm{~mm}$ diameter, $\sim 350 \mu \mathrm{m}$ deep cone is shown in Fig. 5b. A photo of a cone is shown in Fig. 5c. Fig. 6 shows microscope images of a representative large damage site (left) and a typical mitigation cone produced by this process (right). The damage is completely removed. Surface structure created by the circular scan pattern and the individual pulses can be seen in the walls of the cone. The shape of the final cone is not significantly affected by the presence of the damage site (there is a small effect on the depth). 


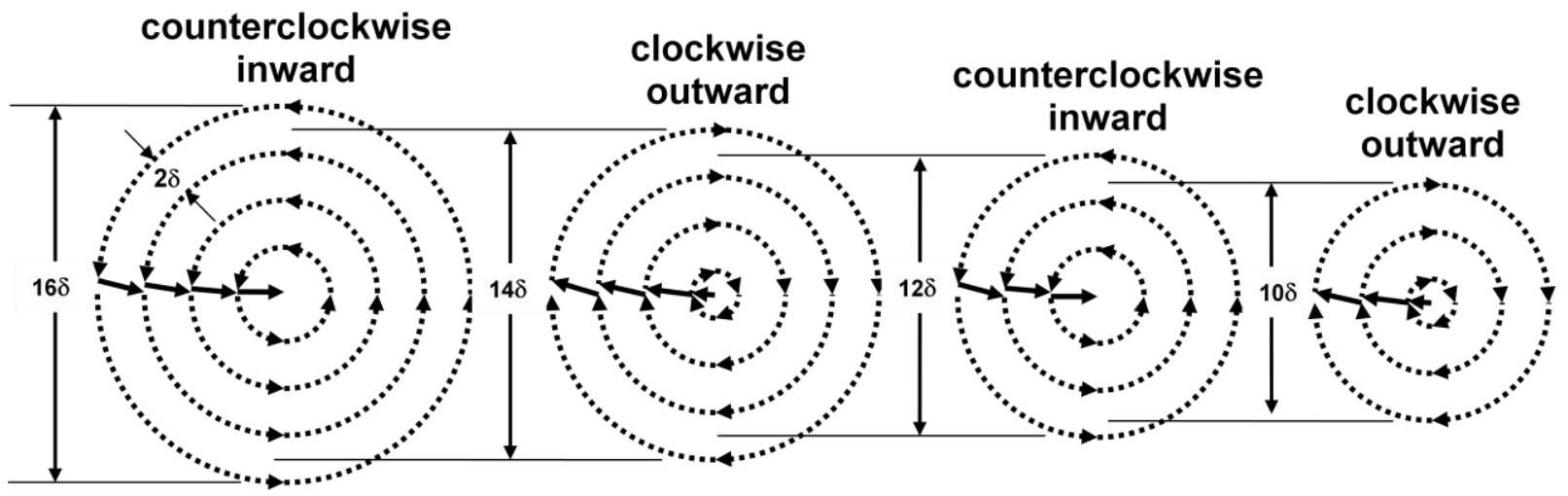

(a)
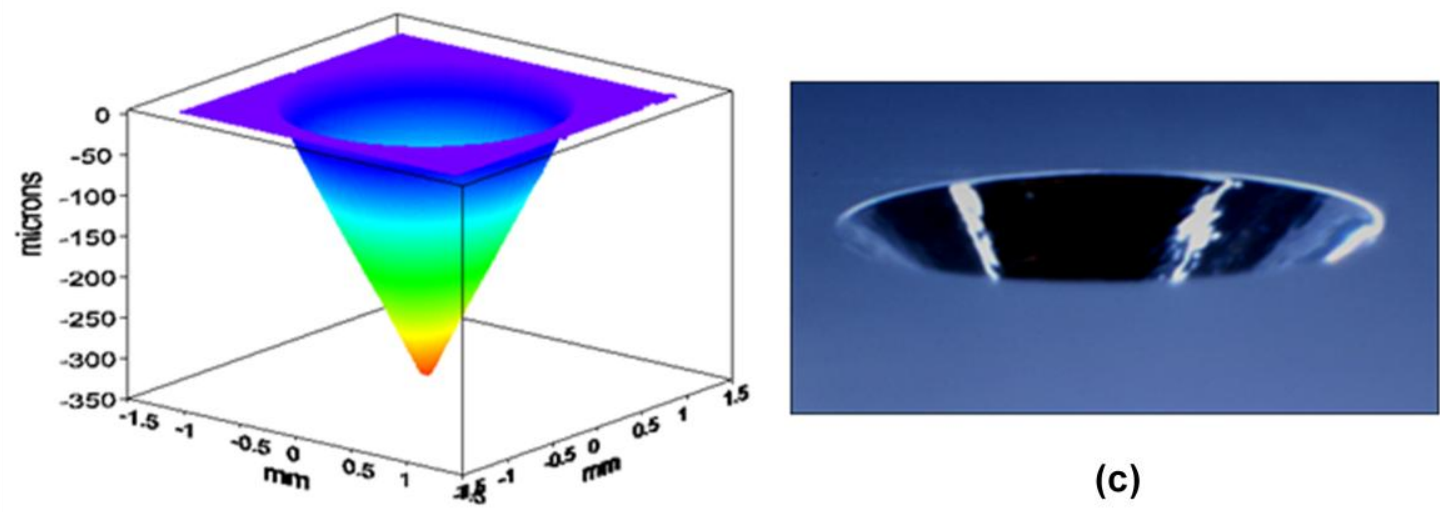

(b)

(c)

Figure 5. (a) Schematic illustrating the scan pattern to produce a mitigation cone. (b) Measured surface profile of a typical cone. (c) Photo of a cone.
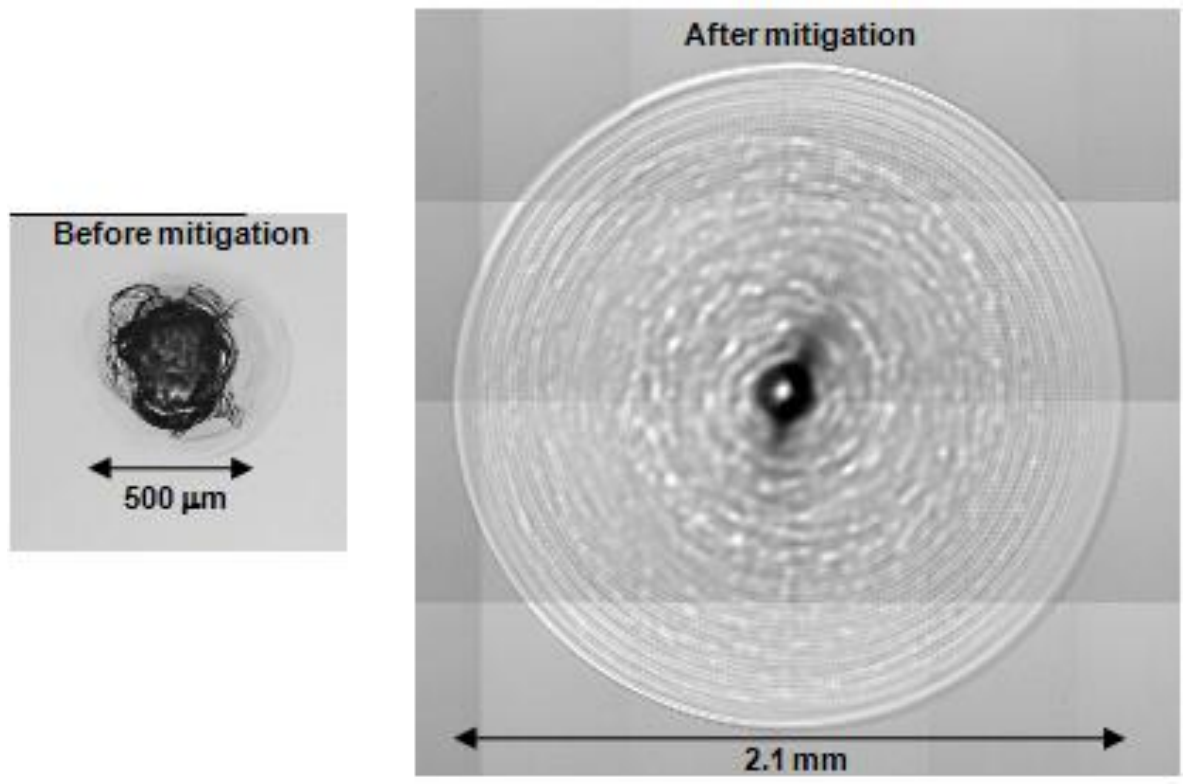

Figure 6. Optical microscope images of a representative large damage site (left) and a typical mitigation cone applied to the damage site (right). 


\section{ISSUES ADDRESSED DURING THE DEVELOPMENT OF THE NEW TECHNIQUE}

Three primary issues were addressed during the development of this new mitigation technique. They were the size and depth of damage sites which could be mitigated, downstream intensification caused by the shape (particularly the raised rim), and management and minimization of re-deposited material. We will describe how these issues were resolved.

\subsection{The size of damage sites that could be mitigated}

The rate at which an optic must be exchanged and serviced is partly determined by the number of shots it can see before growing damage sites become too large to mitigate. As a consequence, it is desirable that the mitigation protocol be able to mitigate as large a damage site as possible. This drives the new mitigation protocol to the largest diameters and depths that can be tolerated within mechanical and optical constraints while still meeting performance requirements. One protocol satisfying these requirements is the $350 \mu \mathrm{m}$ deep cone illustrated in Fig. 7 . The diameter was limited by clipping on the focusing lens for the opto-mechanical configuration of the mitigation facility at the National Ignition Facility (NIF) in Livermore, California. The wall angle was based on observations of laser damage at the perimeter of the site when the wall angle exceeded $24^{\circ}$. It was felt that $18^{\circ}$ gave adequate margin.

To determine the largest damage site that would fit inside this cone, it was necessary to know the upper bound of the aspect ratio of the maximum subsurface crack depth of the damage to its maximum transverse dimension. The upper limit of this aspect ratio was found from measurements on a large sample of damage sites spanning transverse dimensions from $\sim 200 \mu \mathrm{m}$ to $\sim 700 \mu \mathrm{m}$. The subsurface crack depths were measured using a commercial optical coherence tomography system $(\mathrm{OCT})^{11}$ and the transverse dimensions were measured using a microscope. Fitting a cylinder with this aspect ratio (0.61) within the boundaries of the cone showed that it could mitigate damage sites with $\sim 475 \mu \mathrm{m}$ lateral extent and $\sim 275 \mu \mathrm{m}$ depth. This has been confirmed by experiment.

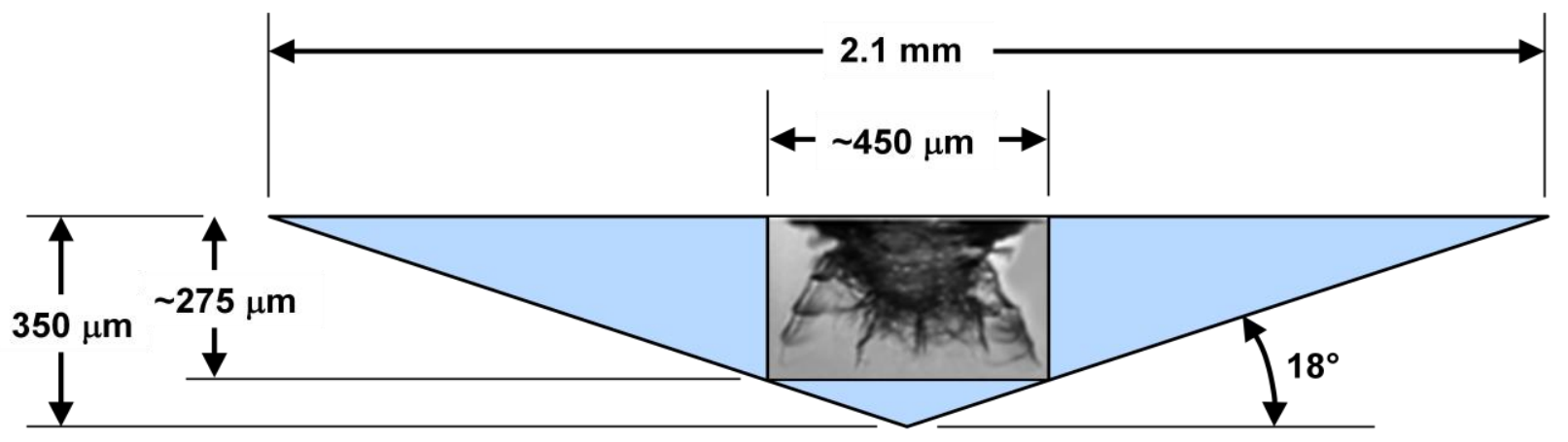

Figure 7. (a) Schematic of a mitigation cone superimposed on a damage site whose aspect ratio was a limiting case based on measurements.

\subsection{Shape effects on downstream intensification}

As previously discussed and illustrated in Fig. 2b, the raised rim surrounding a nominally circular mitigation site will produce an on-axis hot-spot (Fig. 2c) that can be intense enough to damage downstream optical surfaces. The rays producing the hot-spot originate from the outer slopes of the rim. The amount of light focused into the hot-spot scales with the area of the outer slope. In general, larger raised-rims focus more light and produce higher intensifications.

Sectional profiles of the raised-rim produced by the previous and new mitigation techniques are shown in Fig. 8 . It is seen that the rim produced by the new technique is roughly an order-of-magnitude smaller in height and width than that from the previous technique. This is a direct result of thermo-capillary flow being restricted to areas of the order of the $\mathrm{CO}_{2}$ laser spot size in the new technique while the flow in the previous technique took place over areas comparable to the area of the mitigation pit itself.

The resulting on-axis intensifications are compared in Fig. 9. While the previous technique produces an intensification $>5$ times the input light level (the measurement saturated at the highest intensification), the intensification with the new technique remained $<2.5$. The amount of light focused scales with the circumference of the raised-rim as well as its size. The diameter of the $2 \mathrm{~mm}$ cone is $\sim 4 \mathrm{x}$ the diameter of the mitigation pit of the previous technique (see Fig. 1). The measured intensifications are consistent with perimeter and rim size scaling. 
The important practical consequence of the small raised-rim generated with the new technique is that the intensification remains below a level that would damage downstream optical surfaces. This eliminates the need for the "dimpling" step that was required with the previous technique.

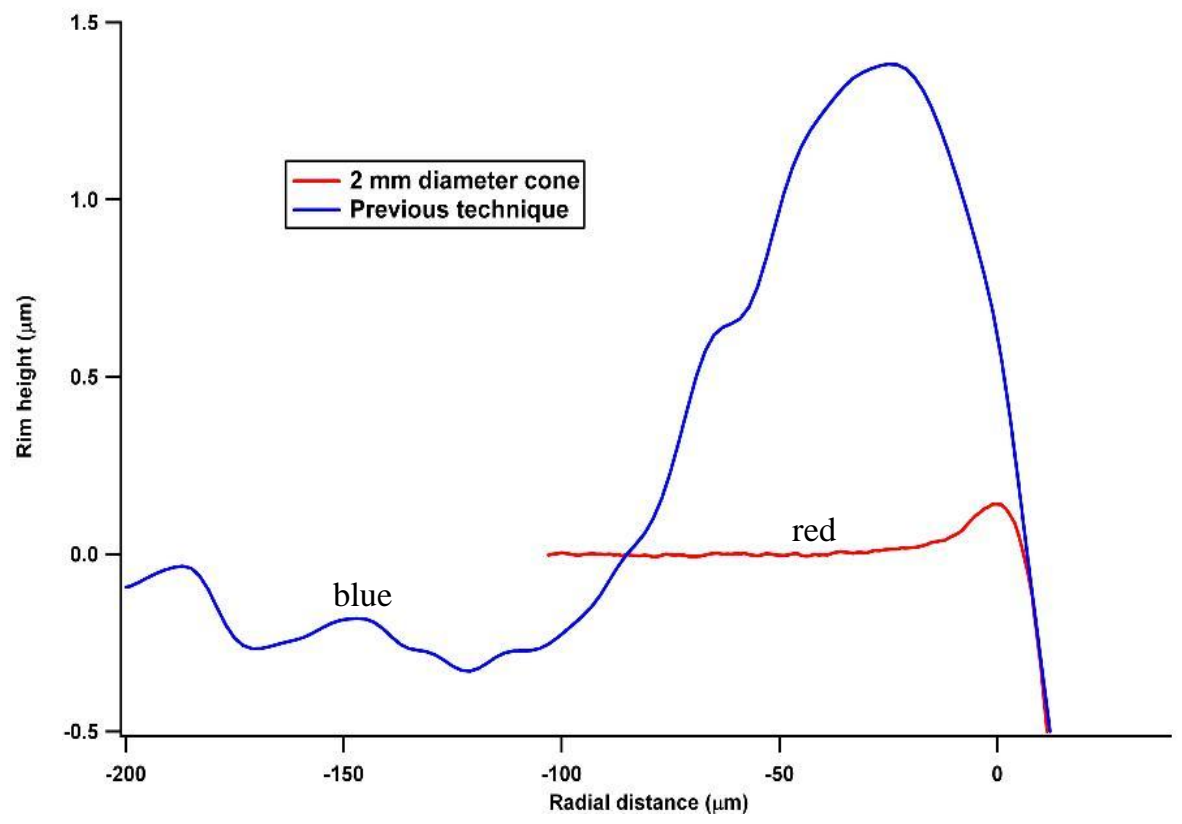

Figure 8. Comparison of the raised-rim produced by the previous (blue) and new (red) mitigation techniques. The edge of the cone is at zero on the radial distance axis.

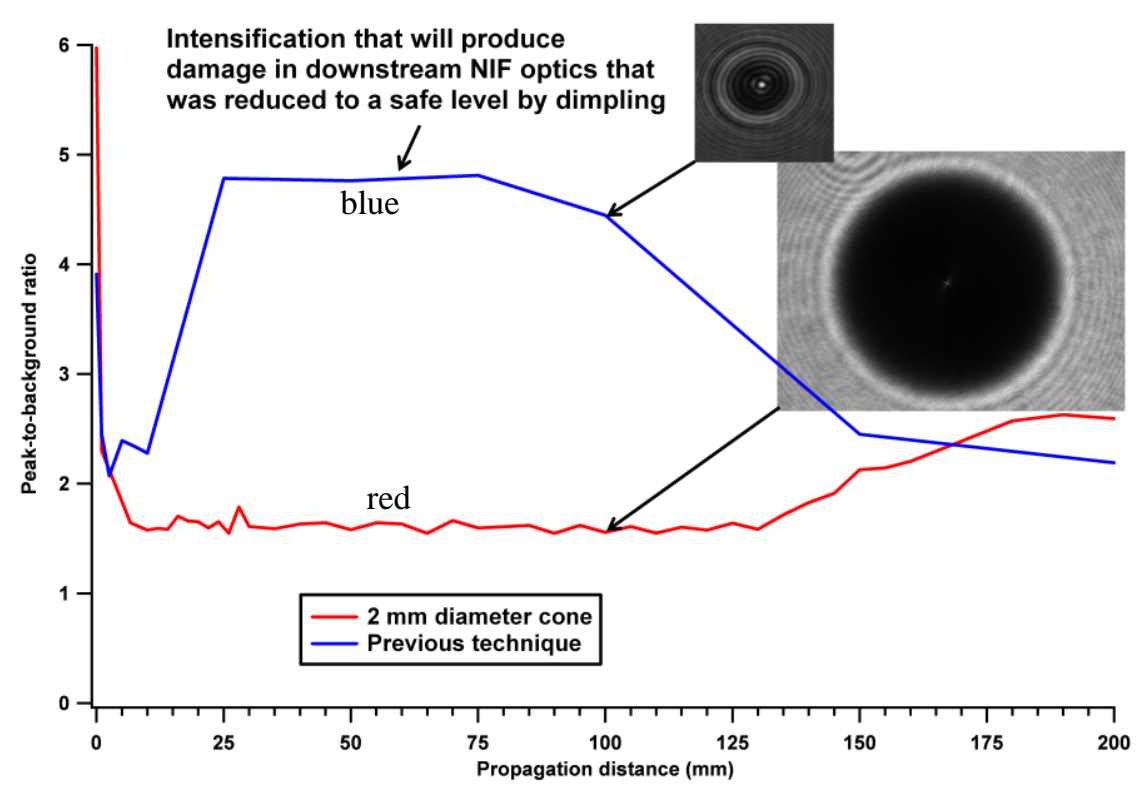

Figure 9. Comparison of the downstream intensification produced by the previous (blue) and new (red) mitigation techniques.

Another mechanism for downstream intensification associated with the conical shape is illustrated schematically in Fig. 10. It shows how the expanding annular wave-front of the light refracted from the cone walls interferes with the incident beam outside the cone. This intensification can exceed a factor of 4 when modulated by local structure in the walls of 
the cone. The key feature of this intensification is that it decreases with the propagation distance as the light is spread over an increasing circumference.

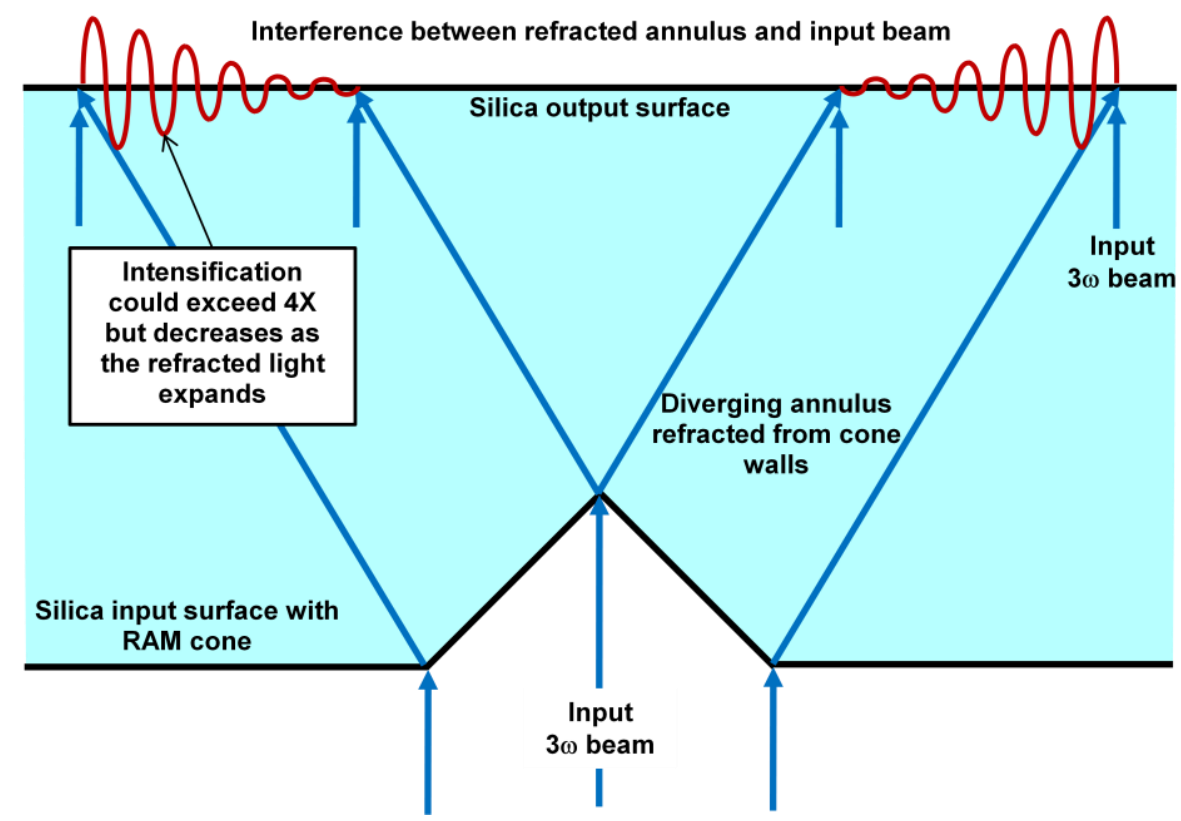

Figure 10. Schematic illustration of the interference between the expanding annular wavefront refracted from the cone wall with the incident beam outside the cone.

This intensification is an issue particularly for mitigation on the input surface of an optic because of the close proximity of the exit surface. The intensification was reduced to an acceptable level at the exit surface by using smaller diameter cones with steeper walls (wall steepness on the input surface does not present the same damage issue as it does on the exit surface). Smaller diameters help because the intensity of the annular wave-front at the exit surface is proportional to the ratio of its initial radius (the mitigation site radius) to its radius at the exit surface. Steeper walls help because the wave-front diverges more rapidly and thus increase the radius at the exit surface. Two shapes found suitable for input surface mitigation are shown in Fig. 11 (one a cone with parabolic walls). These shapes reduce the intensification 10 $\mathrm{mm}$ downstream by $\sim 2 \mathrm{x}$ compared to that from the $2 \mathrm{~mm}$ diameter cone.
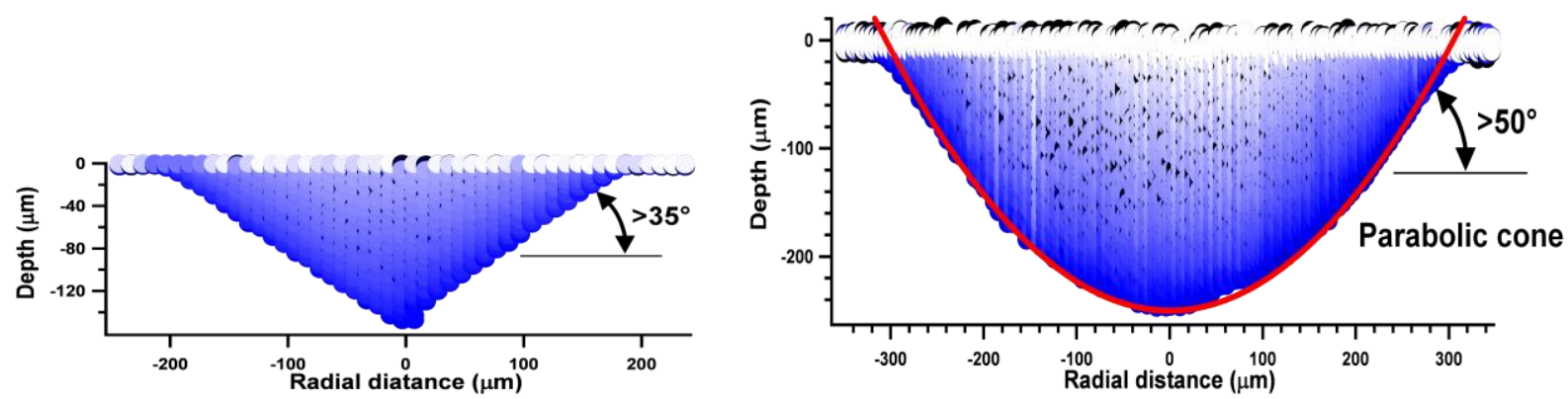

Figure 11. Profiles of mitigation sites which are suitable for input surface mitigation of optics $>1 \mathrm{~cm}$ thick because of their smaller diameters and steeper walls compared to the $2 \mathrm{~mm}$ diameter cone. The surface is at depth $=0$ and negative depths are in the bulk.

\subsection{Re-deposited material}

The primary advantage of the new technique with regard to re-deposit is the high temperature of evaporation (4000K to $5000 \mathrm{~K})$. The resulting high evaporation rate ejects the vapor and condensed nano-particles at high speeds $(\sim 1 \mathrm{~m} / \mathrm{s})$ away from the surface thus significantly reducing the re-deposit. This is graphically illustrated in Fig. 12 which shows the evaporation plume extending several centimeters away from the surface. 
A number of factors affect the amount of re-deposit including the pulse length, the laser power, the PRF, the wall angle of the cone, the scan pattern, and environmental factors such as air flow, humidity, etc. These were optimized to minimize the re-deposit as shown by the scanning electron microscope (SEM) images of Fig. 13. Whereas the re-deposit consists of a fibrous network of nano-particles before optimization (left image and inset of Fig. 13), only a few, well separated nano-particles scattered on the surface around the perimeter of the site remain after optimization (right image of Fig. 13). Before optimization the damage threshold of the re-deposit was $<10 \mathrm{~J} / \mathrm{cm}^{2}$ of $351 \mathrm{~nm}$ light. After optimization it was $>12 \mathrm{~J} / \mathrm{cm}^{2}$.

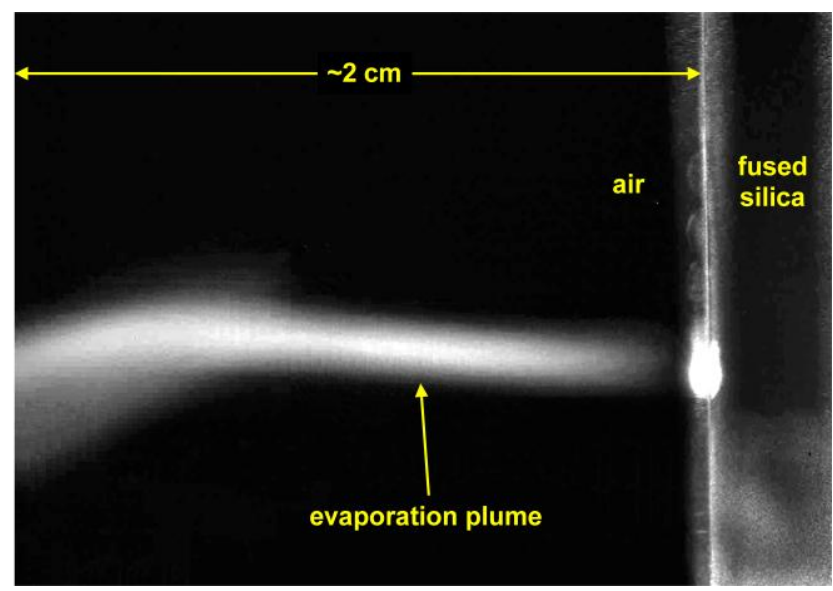

Figure 12. The plume of vapor and condensed nano-particles ejected from the surface during the mitigation process with the new technique.

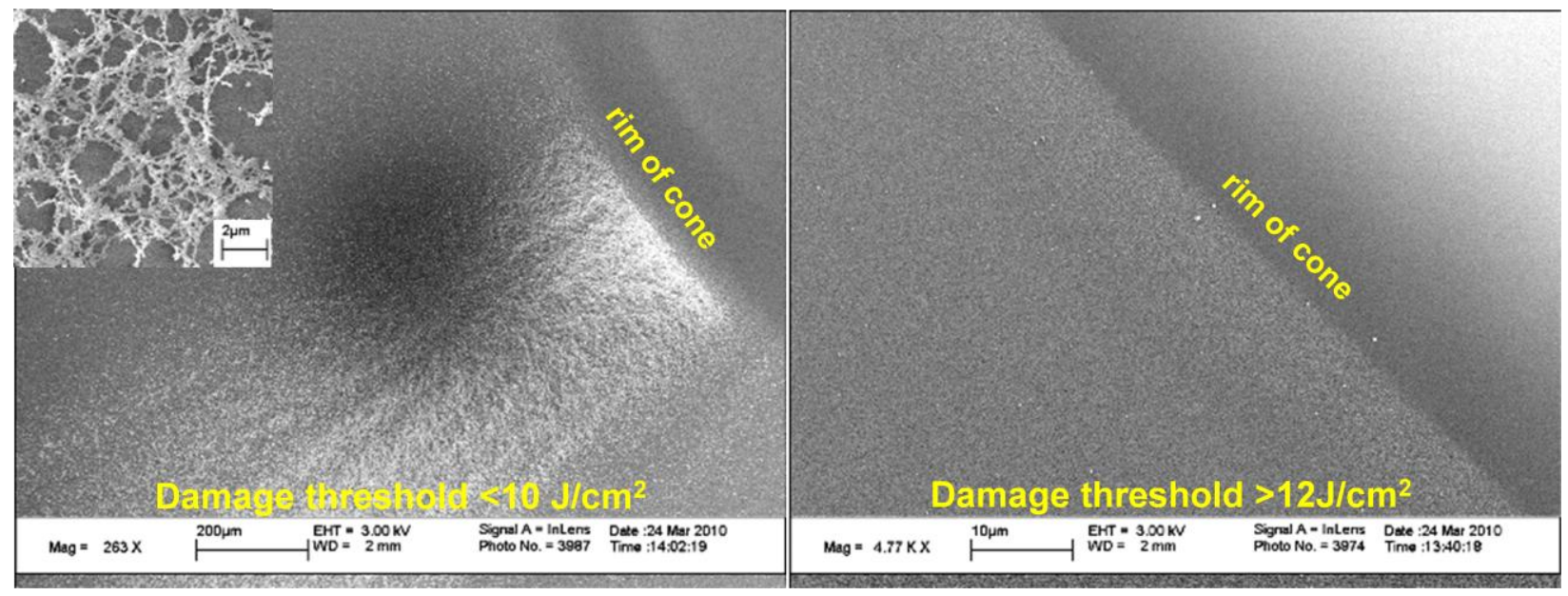

Figure 13. SEM images of the re-deposit before (left) and after (right) in the new mitigation technique.

\section{SUMMARY}

A new evaporative mitigation technique has been developed for laser damage sites on the surfaces of high-value fused silica optics. It eliminates the drawbacks of the previous evaporative technique. The previous technique utilized a 10.6 $\mu \mathrm{m}$ wavelength $\mathrm{CO}_{2}$ laser operating in a $\mathrm{CW}$ mode that heated the entire mitigation area to temperatures allowing thermo-capillary flow. As a consequence, significant downstream intensification was caused by the raised-rim around the site, a large amount of re-deposit on the surface was generated, and a high residual stress was produced. This required additional steps in the process to disrupt the circular symmetry of the raised-rim, to re-melt the re-deposit, and to anneal the stress. In contrast, the new technique operates the $\mathrm{CO}_{2}$ laser in a pulsed mode that limits the thermocapillary flow to an area comparable to the focused spot size. It also allows substantial cooling between pulses. As a 
result, the raised-rim is reduced in size by an order of magnitude, and the downstream intensification is decreased to acceptable levels. The high evaporation temperatures eject material away from the surface at speeds that leaves a negligible amount of re-deposit in the form of nano-particles. The average power with the new technique is an order of magnitude less than that with the previous technique resulting in very low residual stress. Because of these differences, the new technique involves only a single evaporation step compared to the 4-step process of the previous technique that involved optical reconfigurations for two of the steps. The new technique offers versatility in generating difference mitigation shapes. Fig. 14 shows various shapes that have been evaluated.

\section{ACKNOWLEDGMENTS}

This work performed under the auspices of the U.S. Department of Energy by Lawrence Livermore National Laboratory under Contract DE-AC52-07NA27344.

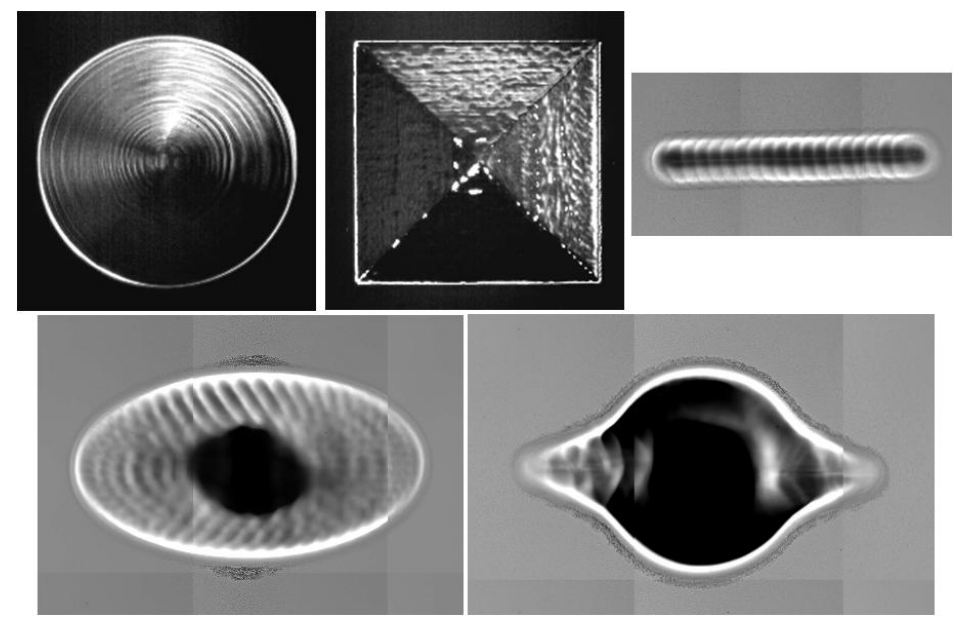

Figure 14. Various shapes produced using the new mitigation technique.

\section{REFERENCES}

[1] Lawrence W. Hrubesh, Mary A. Norton, William A. Molander, Eugene E. Donohue, Stephen M. Maricle, Bernie Penetrante, Raymond M. Brusasco, Walter Grundler, Jim A. Butler, Jeff Carr, R. Hill, Leslie J. Summers, Michael D. Feit, Alexander M. Rubenchik, Michael H. Key, Paul J. Wegner, Alan K. Burnham, Lloyd A. Hackel, and Mark R. Kozlowski, "Methods for mitigating surface damage growth in NIF final optics," Proc. SPIE 4679, 23 (2002).

[2] R. M. Brusasco, B. M. Penetrante, J. A. Butler and L. W. Hrubesh, "Localized $\mathrm{CO}_{2}$ laser treatment for mitigation of $351 \mathrm{~nm}$ damage growth on fused silica", Laser-Induced Damage in Optical Materials: 2001, Proc. SPIE 4679, 40-47 (2002).

[3] Alan K. Burnham, Lloyd A. Hackel, Paul J. Wegner, Thomas G. Parham, Lawrence W. Hrubesh, Bernie M. Penetrante, Pamela K. Whitman, Stavros G. Demos, Joseph A. Menapace, Michael J. Runkel, Michael J. Fluss, Michael D. Feit, Michael H. Key, and Thomas A. Biesiada, "Improving 351-nm damage performance of largeaperture fused silica and DKDP optics," Proc. SPIE 4679, 173 (2002).

[4] P. Bouchut, L. Delrive, D. Decruppe and P. Garrec, "Local re-fusion of silica, by a continuous CO2 laser, for the mitigation of laser damage growth," Proc. SPIE 5252, 122 (2004).

[5] Isaac L. Bass, Gabriel M. Guss, and Richard P. Hackel, "Mitigation of laser damage growth in fused silica with a galvanometer scanned $\mathrm{CO}_{2}$ laser," Proc. SPIE 5991, 59910C, (2005).

[6] Isaac L. Bass, Vaughn G. Draggoo, Gabriel M. Guss, Richard P. Hackel, and Mary A. Norton, "Mitigation of laser damage growth in fused silica NIF optics with a galvanometer scanned $\mathrm{CO}_{2}$ laser," Proc. SPIE 6261, 62612A (2006). 
[7] Adams, J. J., Bude, J. D., Bolourchi, M., Guss, G. M., Matthews, M. J., Nostrand, M. C., "Results of applying a non-evaporative mitigation technique to laser-initiated surface damage on fused silica," Proc. SPIE 7842, to be published, (2010)

[8] Gabe Guss, Isaac Bass, Vaughn Draggoo, Richard Hackel, Steve Payne, Mark Lancaster, and Paul Mak, "Mitigation of growth of laser initiated surface damage in fused silica using a 4.6 $\mu \mathrm{m}$ wavelength laser," Proc. SPIE 6403, 64030M-1 (2006).

[9] Yang, S. T., Matthews, M. J., Elhadj, S. Cooke, D.J., Guss, G. M., Draggoo, V. G., Wegner, P. J., "Comparing the use of mid-infrared versus far-infrared lasers for mitigating damage growth on fused silica," Proc. SPIE 7842, to be published (2010).

[10] T. R. Anthony and H. E. Cline, "Surface rippling induced by surface-tension gradients during laser surface melting and alloying," Journal of Applied Physics 48(9), 3888-3894 (1977).

[11] Guss, G. M., Bass, I. L., Hackel, R. P., Mailhiot, C., and Demos, S. G., "In situ monitoring of surface post processing in large aperture fused silica optics with optical coherence tomography," Applied Optics 47(25), 4569 (2008). 